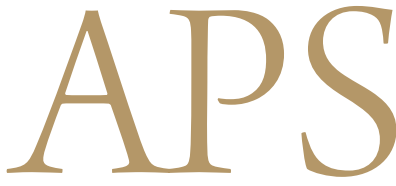

Archives of Plastic Surgery

\title{
Adequacy of sentinel lymph node biopsy in malignant melanoma of the trunk and extremities: Clinical observations regarding prognosis
}

\author{
Yong Chan Bae ${ }^{1,2}$, Dae Kyun Jeong ${ }^{1}$, Kyoung Hoon Kim ${ }^{3}$, Kyung Wook Nam ${ }^{1}$, Geon Woo Kim ${ }^{1}$, \\ Hoon Soo Kim ${ }^{4}$, Su Bong Nam ${ }^{1}$, Seong Hwan Bae ${ }^{1}$ \\ ${ }^{1}$ Department of Plastic and Reconstructive Surgery, Pusan National University School of Medicine, Busan; ${ }^{2}$ Biomedical Research Institute, \\ Pusan National University Hospital, Busan; ${ }^{3}$ Brand Plastic Surgery, Busan; ${ }^{4}$ Department of Dermatology, Pusan National University School \\ of Medicine, Busan, Korea
}

Background Methods for identifying local lymph node metastasis in malignant melanoma include sentinel lymph node biopsy (SLNB) and lymph node dissection (LND). In particular, SLNB has been widely used in recent years. This study aimed to retrospectively confirm the adequacy of the current indication criteria for SLNB by applying those criteria to a mixed group of patients who previously received SLNB and LND.

Methods This study included 77 patients with malignant melanoma of the extremities and trunk who were monitored for $>24$ months. The patients were classified according to whether the current indication criteria for SLNB were applicable. The sentinel lymph nodes were evaluated for each group. Patients for whom the indication criteria for SLNB and LND were applicable were analyzed according to whether SLNB or LND was performed. Finally, the outpatient records of these patients were reviewed to evaluate recurrence, metastasis, and prognosis.

Results Of the 77 patients, SLNB was indicated according to the current criteria in 60 cases. Among the 60 patients for whom SLNB was indicated, 35 survived the follow-up period disease-free, 21 died during the follow-up period, and four experienced metastasis. The 17 patients for whom SLNB was not indicated had no recurrence or metastasis.

Conclusions Patients for whom SLNB was not indicated had no recurrence or metastasis. In cases where SLNB is indicated, the possibility of metastasis and recurrence may be high even if SLNB is negative or LND is performed, so more aggressive treatment and careful follow-up are crucial.

Keywords Melanoma / Lymph / Biopsy / Skin neoplasms / Prognosis
Correspondence: Seong Hwan Bae Department of Plastic and Reconstructive Surgery, Pusan National University School of Medicine, 179 Gudeok-ro, Seo-gu, Busan 49241, Korea Tel: +82-51-240-7396 Fax: +82-51-243-9405 E-mail:denev96@gmail.com

This work was supported by a Clinical Research Grant from Pusan National University Hospital in 2020.

Received: July 20, 2019 • Revised: September 25, 2019 • Accepted: October 18, 2019

pISSN: 2234-6163 • elSSN: 2234-6171 • https://doi.org/10.5999/aps.2019.00934• Arch Plast Surg 2020;47:42-48

\section{INTRODUCTION}

Malignant melanoma is the most aggressive skin cancer and has a high potential for early metastasis. Therefore, accurate staging and appropriate management are essential for increasing the survival rate. The most crucial factors in evaluating the prognosis of malignant melanoma are the thickness of the lesion and local lymph node metastasis [1-4]. In the past, elective lymph 
node dissection (LND) was performed to confirm local lymph node metastasis. However, after Morton et al. [5] reported the use of sentinel lymph node biopsy (SLNB), SLNB has been widely performed due to its relative simplicity and accurate results. In the treatment of malignant melanoma, SLNB is indicated for patients with melanomas $>1 \mathrm{~mm}$ in thickness and patients with thin melanomas ( $\leq 1 \mathrm{~mm}$ in thickness) that demonstrate high-risk features, including ulceration and/or a high mitotic rate $[6-8]$.

In Asian countries, including Korea, the prevalence of malignant melanoma is lower than in Western countries, and the most common subtype is the acral lentiginous type, which is known to have a relatively poor prognosis [9-12]. Furthermore, many studies on sentinel lymph node status and patient survival outcomes have been published in Western countries, but studies published in Asian countries are rare; thus, more studies on sentinel lymph node status in Asian countries are needed.

SLNB was first introduced in the sixth edition of the American Joint Committee on Cancer Staging Manual, which was published in 2002, and information regarding the indications for SLNB was included starting with the seventh edition, published in 2009. Therefore, until 2009, SLNB and LND were commonly used together to confirm local lymph node metastasis. The authors similarly used a combination of SLNB and LND to confirm local lymph node metastasis before 2009; thereafter, a gradual transition was made towards SLNB. Therefore, patients treated by the authors in the past constituted a mixed group of patients who underwent SLNB, LND, or neither procedure. In light of this fact, the authors aimed to confirm the adequacy of the current indication criteria for SLNB by applying these criteria to this mixed group of patients.

\section{METHODS}

\section{Participants}

Approval from the ethics committee of our institution (H-1909010-083) was obtained prior to conducting this study. At the start of this retrospective study, 110 consecutive patients who were diagnosed with malignant melanoma and underwent surgical treatment at Pusan National University Hospital between September 2000 and August 2015 were screened. Among these patients, 14 patients with head and neck melanoma were excluded due to difficulty locating a sentinel lymph node.

\section{Procedures}

In this study, wide local excision margins were adopted, with margins determined based on the Breslow thickness. The surgical margin was $0.5 \mathrm{~cm}$ for in situ melanoma, $1 \mathrm{~cm}$ for lesions $\leq 1$ $\mathrm{mm}$ in thickness, $2 \mathrm{~cm}$ for lesions $1.01-2.00 \mathrm{~mm}$ in thickness, and $3 \mathrm{~cm}$ for lesions $>2.00 \mathrm{~mm}$ in thickness.

The surgical margins of subungual melanomas were determined largely using the Breslow thickness, as described above. For subungual melanomas of the fingers, if the determined surgical margins were confined to the distal interphalangeal (DIP) joint level, DIP joint-level amputation was performed. If the surgical margins exceeded the DIP joint level but were confined to the proximal interphalangeal (PIP) joint level, PIP joint-level amputation was performed. Similarly, for subungual melanomas of the toes, amputation was performed at either the level of the PIP joints or the metatarsophalangeal joints. Melanomas of the foot (except the toes), forearm, and leg were generally treated with a wide excision margin. Surgical wounds were covered with skin grafts or local flaps. To facilitate weight-bearing, free flaps were used for reconstruction of the sole.

Primary melanomas of the trunk were generally excised with more liberal margins. The resulting surgical wounds were covered with skin grafts or, in some areas, closed with wide undermining and a large advancement flap.

On either the day before or the morning of the operation, patients underwent lymphoscintigraphy at the nuclear medicine department to determine the location of the sentinel nodes. Patients with malignant melanomas were injected with $0.2 \mathrm{~mL}$ of ${ }^{99 \mathrm{~m}} \mathrm{Tc}$ phytate. In the operating room, a gamma probe (Neoprobe GDS; Devicor Medical Products, Inc., Cincinnati, OH, USA) was used to detect radioactivity in the sentinel lymph nodes. The sentinel lymph nodes were removed and sent to the pathology department.

The authors first examined the histopathological results and lesion thickness of 77 patients, then assessed regional lymph node metastasis or distant metastasis using imaging, including regional computed tomography, lymphoscintigraphy, and positron emission tomography-computed tomography. Based on the collected information, patients were classified according to whether the current indication criteria for SLNB were applicable. Patients for whom SLNB was indicated according to the current criteria were classified according to whether SLNB had been performed and, if the results of SLNB were positive, whether LND was performed. Patients for whom SLNB was not indicated were also classified according to whether SLNB had been performed. Finally, the outpatient records of these patients were reviewed with regard to recurrence, metastasis, and prognosis.

\section{Statistical analysis}

Statistical analysis of the data collected was performed using SPSS version 22.0 (IBM Corp., Armonk, NY, USA). The chi- 
square test was used to assess the associations between categorical data and mortality. When the expected frequency was less than 5, the Fisher exact test was used. A P-value of less than 0.05 was considered to indicate statistical significance.

\section{RESULTS}

Of the 96 patients for whom outpatient follow-up results were investigated, 77 patients who either died of melanoma during the follow-up period or whose follow-up period was 24 months or longer were included in this study. The follow-up period ranged from 24 to 122 months, with an average of 44.5 months. Of the 77 patients, $26(33.8 \%)$ had nodular melanomas, 46 (59.7\%) had acral lentiginous melanomas, and five (6.5\%) had superficial spreading melanomas. Among them, 69 patients underwent either SLNB or LND to confirm lymph node metastasis. The sentinel lymph nodes were found in the inguinal region in 40 cases (58.0\%), in the axillary region in 19 cases (27.5\%), in the popliteal region in 5 cases $(7.2 \%)$, and in both the popliteal and inguinal regions in 5 cases $(7.2 \%)$ (Table 1$)$.

SLNB was indicated in 60 patients, and was not indicated in the remaining 17. Of the 60 patients for whom SLNB was indicated, five patients declined the procedure, and a biopsy was not obtained. Of the five patients who declined SLNB, three experienced metastasis and died during the follow-up period, while two patients survived the follow-up period disease-free. Of the remaining 55 patients, SLNB was performed in 35 and LND was performed in the other 20 . Of the 35 patients who underwent SLNB, 18 had a negative result for the sentinel lymph node, so additional surgery was not performed. Of these $18 \mathrm{pa}-$ tients, two patients died, two received adjuvant therapy for metastasis, and 14 survived the follow-up period disease-free.

Of the 17 patients with positive SLNB, one patient refused LND and died of recurrence. Eight patients had positive LND, of whom four patients died, two underwent adjuvant therapy for metastasis, and two survived disease-free throughout the follow-up period. Of the eight patients with negative LND, one died of recurrence and seven survived the follow-up period disease-free. Of the 20 patients who underwent LND without SLNB, nine had a positive result, five died, and four survived the follow-up period disease-free. Of the $11 \mathrm{LND}$-negative patients, five died of recurrence during the follow-up period, and six survived disease-free. In summary, of the 60 patients for whom SLNB was indicated, 35 survived the follow-up period diseasefree, 21 died during follow-up, and four received chemotherapy or adjuvant therapy for metastasis.

Of the 17 patients for whom SLNB was not indicated, 14 had negative SLNB. All 17 patients for whom SLNB was not indi-

\begin{tabular}{|c|c|}
\hline Characteristic & No. $(\%)$ \\
\hline \multicolumn{2}{|l|}{ Sex } \\
\hline Male & $27(35.1)$ \\
\hline Female & $50(64.9)$ \\
\hline \multicolumn{2}{|l|}{ Age (yr) } \\
\hline$<39$ & $6(7.8)$ \\
\hline $40-49$ & $9(11.7)$ \\
\hline $50-59$ & $12(15.6)$ \\
\hline $60-69$ & $26(33.8)$ \\
\hline $70-79$ & $18(23.4)$ \\
\hline$\geq 80$ & $6(7.8)$ \\
\hline \multicolumn{2}{|l|}{ Subtype } \\
\hline Nodular & $26(33.8)$ \\
\hline Acral & $46(59.7)$ \\
\hline Superficial & $5(6.5)$ \\
\hline \multicolumn{2}{|l|}{ Primary site } \\
\hline Upper extremity & $16(20.8)$ \\
\hline Hand & $11(14.3)$ \\
\hline Finger (subungual) & $11(14.3)$ \\
\hline Finger (except subungual) & 0 \\
\hline Other & 0 \\
\hline Other & $5(6.5)$ \\
\hline Lower extremity & $53(68.8)$ \\
\hline Foot & $41(53.2)$ \\
\hline Toes (subungual) & $4(5.2)$ \\
\hline Toes (except subungual) & $3(3.9)$ \\
\hline Other & $34(44.2)$ \\
\hline Other & $12(15.6)$ \\
\hline Trunk & 8 (10.4) \\
\hline \multicolumn{2}{|l|}{ Thickness (mm) } \\
\hline In situ & $13(16.9)$ \\
\hline$\leq 1$ & $4(5.2)$ \\
\hline $1.01-2.00$ & $12(15.6)$ \\
\hline $2.01-4.00$ & $22(28.6)$ \\
\hline$\geq 4.01$ & $26(33.8)$ \\
\hline \multicolumn{2}{|l|}{ Stage } \\
\hline 0 & 13 (16.9) \\
\hline I & $15(19.5)$ \\
\hline$\|$ & $26(33.8)$ \\
\hline III & $17(22.1)$ \\
\hline IV & $6(7.8)$ \\
\hline \multicolumn{2}{|l|}{ Location of sentinel lymph node(s) } \\
\hline Axillary & $19(27.5)$ \\
\hline Inguinal & $40(58.0)$ \\
\hline Popliteal & $5(7.2)$ \\
\hline Inguinal and popliteal & $5(7.2)$ \\
\hline \multicolumn{2}{|l|}{ Clark level } \\
\hline I & $13(16.9)$ \\
\hline$\|$ & $9(11.7)$ \\
\hline III & $9(11.7)$ \\
\hline IV & $29(37.7)$ \\
\hline V & $17(22.1)$ \\
\hline
\end{tabular}

cated had no recurrence or metastasis, regardless of whether they underwent lymph node biopsy (Fig. 1).

The chi-square test of independence showed no statistically significant difference by sex in terms of whether SLNB was indi- 


\section{Fig. 1. Patient classification algorithm}

The authors classified patients according to whether the current indication criteria for sentinel lymph node biopsy (SLNB) were applicable whether SLNB was performed if so, and whether lymph node dissection (LND) was performed if SLNB results were positive. Patients for whom SLNB was not indicated were also classified according to whether they underwent SLNB. LN evaluation, SLNB or LND.

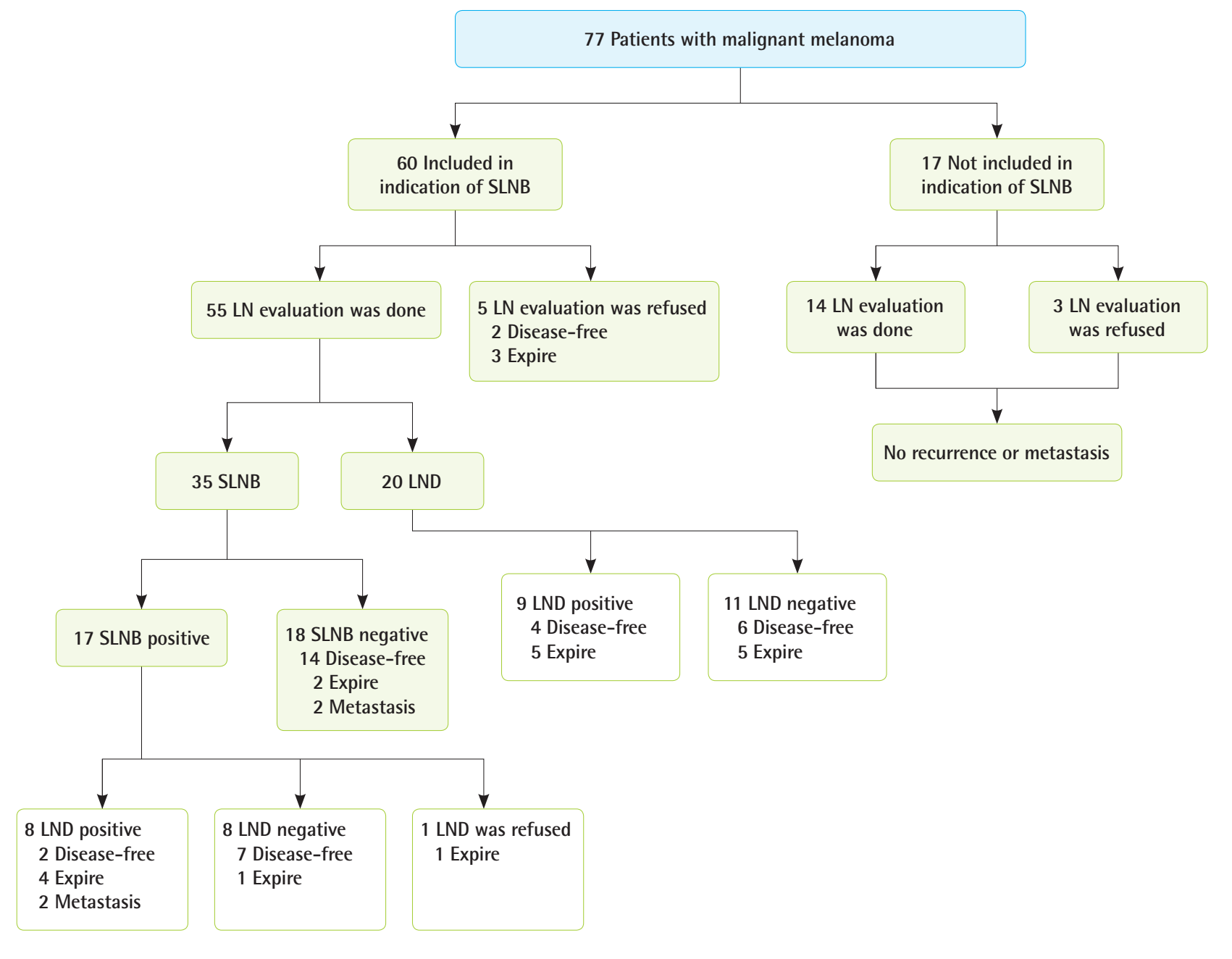

cated $(\mathrm{P}=0.982)$. The Fisher exact test showed no statistically significant difference in terms of whether SLNB was indicated according to age $(P=0.191)$, subtype $(P=0.075)$, primary site $(\mathrm{P}=0.753)$, or sentinel lymph node location $(\mathrm{P}=0.382)$. However, statistically significant relationships were found between thickness $(P=0.000)$, stage $(P=0.000)$, and Clark level $(P=0.000)$ and whether SLNB was indicated (Table 2).

\section{DISCUSSION}

In this study, we retrospectively investigated the prognosis of patients with malignant melanoma of the trunk and extremities who underwent a combination of SLNB and LND to determine the appropriateness of the current indication criteria for SLNB. In the analysis of demographic information, no statistically sig-
Table 2. Demographic and clinical information of the patients analyzed in this study

\begin{tabular}{|lc|}
\hline Demographic and clinical information & P-value \\
\hline Sex & 0.982 \\
Age distribution & 0.191 \\
Subtype & 0.075 \\
Primary site & 0.753 \\
Sentinel lymph node location & 0.382 \\
Breslow thickness & $0.000^{\text {a) }}$ \\
Stage & $0.000^{a)}$ \\
Clark level & $0.000^{a)}$ \\
\hline a)Statistically significant; $P<0.05$. & \\
\hline
\end{tabular}

nificant differences with regard to sex, age distribution, sentinel lymph node location, or subtype were found between patients for whom SLNB was indicated and those for whom SLNB was 
not indicated. However, statistically significant differences were noted with regard to the Breslow thickness, stage, and Clark level. These parameters are thought to be directly associated with whether SLNB is indicated.

The most common subtype of melanoma differs between Asian and Western populations. Roh et al. [12] reported that acral lentiginous melanoma accounted for $65 \%$ of 40 Korean melanoma patients. Lee et al. [13] reported that the rate of acral lentiginous melanoma was significantly different between Asians and Caucasians living in Singapore (56\% and 14\%, respectively). In two other studies of Korean melanoma patients, 58 of 100 patients and 58 of 113 patients had acral lentiginous melanoma (58\% and 51.3\%, respectively) $[14,15]$. The prevalence of acral lentiginous melanoma in the present study was $59.7 \%$, which is similar to that of Asian patients in the previous studies.

There is controversy as to whether SLNB is necessary if SLNB is not indicated. In general, the probability of lymph node metastasis in cases of malignant melanoma increases with the thickness of the lesion. Rousseau et al. [6] reported 1,375 patients with malignant melanoma who underwent SLNB, of whom $4 \%$ displayed sentinel lymph node metastasis when the lesion thickness was $>1 \mathrm{~mm}, 12 \%$ when it was $1-2 \mathrm{~mm}, 28 \%$ when $2-4 \mathrm{~mm}$, and $44 \%$ when $>4 \mathrm{~mm}$. Moreover, Rousseau et al. [6] suggested that SLNB was unnecessary unless indicated. In the present study, all patients for whom SLNB was not indicated displayed no lymph node metastasis and were disease-free at the end of the follow-up period. These results are lower than those of Rousseau et al. Therefore, based on the present study, it can be considered unnecessary to perform SLNB in cases other than those in which it is indicated.

False negatives take place when cancer recurs despite negative biopsy results for SLNB and LND. False-negative results are likely to occur either when malignant cells fail to reach the sentinel lymph node by the time of the biopsy because the migration of cancer cells through the lymph duct is very slow, or when malignant cells are not detected in the pathologic examination. In a retrospective review of 1,287 melanoma patients, Carlson et al. [16] reported recurrence in 133 (12.5\%) patients who were sentinel lymph node-negative. In a study of 846 patients, Yee et al. [17] reported that 22 (13.2\%) of 167 patients with negative SLNB experienced recurrence. In the present study, 18 of the 35 patients who underwent SLNB displayed negative SLNB findings. Two of these patients died, and two patients were treated for recurrence at follow-up, so the false-negative rate was $22.2 \%$. This suggests that even if SLNB is negative, the cancer is more likely to recur than has been reported in previous studies.
Wagner et al. [18] reported a $7 \%$ to $33 \%$ possibility of finding additional lymph node metastasis when performing LND in cases with positive SLNB. In addition, according to Morton et al. [19], therapeutic LND may increase the survival rate in patients with positive SLNB. In the present study, another metastatic lymph node was found in $50 \%$ of patients who underwent additional LND, which is a higher rate than that found in previous studies. Therefore, the authors agree that additional therapeutic LND should be performed in SLNB-positive patients.

In Koreans, the most common location of sentinel lymph nodes is the femoral region because the lower limb is the most common site of melanoma in this population. In a study conducted by Thompson et al. [20], 13 (0.31\%) of 4,260 patients with melanoma below the knee had popliteal lymph node metastasis, and 17 (7.2\%) of the 236 patients who underwent lymphoscintigraphy had a popliteal lymph node as a sentinel node. According to Menes et al. [21], 10 (9\%) of 106 patients with malignant melanoma of the lower extremities reported drainage to the popliteal lymph node. In a Korean study, Kim et al. [22] suggested that popliteal lymph nodes, as well as inguinal lymph nodes, should be identified preoperatively because popliteal lymph nodes may test positive, albeit at a low frequency. In the present study, sentinel lymph nodes were found only in the popliteal area in three cases (5\%) and in both the popliteal and inguinal areas in five cases (7\%). Thus, overall, popliteal lymph nodes were identified as sentinel lymph nodes in $12 \%$ of cases, which is higher than the frequency found previously. Therefore, we consider it appropriate to identify popliteal lymph nodes when treating malignant melanoma of the lower extremities, especially in Asian populations.

In the present study, we evaluated patients with malignant melanomas of the trunk and extremities who underwent SLNB and LND. In these patients, the current indication criteria for SLNB were used, patients were categorized accordingly, and their prognoses were examined. We attempted to retrospectively determine the appropriateness of the current indication criteria for SLNB. This method has not been attempted in other studies and may serve as a basis for confirming the clinical relevance of the SLNB indication criteria. However, this study was limited by its relatively small sample size and short follow-up period.

In conclusion, patients for whom SLNB was not indicated according to the current criteria did not have recurrence or metastasis, so we considered it unnecessary to perform SLNB. In cases for which SLNB is indicated, more aggressive treatment and careful follow-up are crucial because the possibility of metastasis and recurrence may be high even if SLNB is negative or LND is performed. 


\section{NOTES}

\section{Conflict of interest}

No potential conflict of interest relevant to this article was reported.

\section{Ethical approval}

The study was approved by the Institutional Review Board of Pusan National University Hospital (IRB No. H-1909-010083) and performed in accordance with the principles of the Declaration of Helsinki. Written informed consents were obtained.

\section{Author contribution}

Conceptualization: Bae YC. Data curation: Nam KW. Formal analysis: Nam KW. Funding acquisition: Nam SB. Methodology: Kim HS. Project administration: Kim KH. Visualization: Kim GW. Writing - original draft: Bae YC, Jeong DK. Writing review \& editing: Bae YC, Bae SH. Approval of final manuscript: all authors.

\section{ORCID}

Yong Chan Bae https://orcid.org/0000-0002-0268-4667 Dae Kyun Jeong https://orcid.org/0000-0002-6379-2629 Kyoung Hoon Kim https://orcid.org/0000-0002-5736-9365 Kyung Wook Nam https://orcid.org/0000-0002-2188-8681 Geon Woo Kim https://orcid.org/0000-0002-5467-9480 Hoon Soo Kim https://orcid.org/0000-0002-7649-1446 Su Bong Nam https://orcid.org/0000-0003-2718-2230 Seong Hwan Bae https://orcid.org/0000-0002-7203-8978

\section{REFERENCES}

1. Gershenwald JE, Thompson W, Mansfield PF, et al. Multiinstitutional melanoma lymphatic mapping experience: the prognostic value of sentinel lymph node status in 612 stage I or II melanoma patients. J Clin Oncol 1999;17:976-83.

2. Balch CM, Soong SJ, Gershenwald JE, et al. Prognostic factors analysis of 17,600 melanoma patients: validation of the American Joint Committee on Cancer melanoma staging system. J Clin Oncol 2001;19:3622-34.

3. Morton DL, Wanek L, Nizze JA, et al. Improved long-term survival after lymphadenectomy of melanoma metastatic to regional nodes: analysis of prognostic factors in 1134 patients from the John Wayne Cancer Clinic. Ann Surg 1991; 214:491-9.

4. McMasters KM, Chao C, Wong SL, et al. Interval sentinel lymph nodes in melanoma. Arch Surg 2002;137:543-7.
5. Morton DL, Wen DR, Wong JH, et al. Technical details of intraoperative lymphatic mapping for early stage melanoma. Arch Surg 1992;127:392-9.

6. Rousseau DL Jr, Ross MI, Johnson MM, et al. Revised American Joint Committee on Cancer staging criteria accurately predict sentinel lymph node positivity in clinically node-negative melanoma patients. Ann Surg Oncol 2003; 10:569-74.

7. Ali-Salaam P, Ariyan S. Lymphatic mapping and sentinel lymph node biopsies. Clin Plast Surg 2000;27:421-9.

8. Balch CM, Gershenwald JE, Soong SJ, et al. Final version of 2009 AJCC melanoma staging and classification. J Clin Oncol 2009;27:6199-206.

9. Johnson DS, Yamane S, Morita S, et al. Malignant melanoma in non-Caucasians: experience from Hawaii. Surg Clin North Am 2003;83:275-82.

10. Qiu D, Marugame T. Comparison of time trends in skin cancer incidence (1973-97) in East Asia, Europe and USA, from cancer incidence in five continents vol. IV-VIII. Jpn J Clin Oncol 2008;38:234-6.

11. Socrier Y, Lauwers-Cances V, Lamant L, et al. Histological regression in primary melanoma: not a predictor of sentinel lymph node metastasis in a cohort of 397 patients. Br J Dermatol 2010;162:830-4.

12. Roh MR, Kim J, Chung KY. Treatment and outcomes of melanoma in acral location in Korean patients. Yonsei Med J 2010;51:562-8.

13. Lee HY, Chay WY, Tang MB, et al. Melanoma: differences between Asian and Caucasian patients. Ann Acad Med Singapore 2012;41:17-20.

14. Nam KW, Bae YC, Bae SH, et al. Analysis of the clinical and histopathological patterns of 100 consecutive cases of primary cutaneous melanoma and correlation with staging. Arch Plast Surg 2015;42:746-52.

15. Nam KW, Bae YC, Nam SB, et al. Characteristics and treatment of cutaneous melanoma of the foot. Arch Plast Surg 2016;43:59-65.

16. Carlson GW, Page AJ, Cohen C, et al. Regional recurrence after negative sentinel lymph node biopsy for melanoma. Ann Surg 2008;248:378-86.

17. Yee VS, Thompson JF, McKinnon JG, et al. Outcome in 846 cutaneous melanoma patients from a single center after a negative sentinel node biopsy. Ann Surg Oncol 2005;12:42939.

18. Wagner JD, Corbett L, Park HM, et al. Sentinel lymph node biopsy for melanoma: experience with 234 consecutive procedures. Plast Reconstr Surg 2000;105:1956-66.

19. Morton DL, Cochran AJ, Thompson JF, et al. Sentinel node 
biopsy for early-stage melanoma: accuracy and morbidity in MSLT-I, an international multicenter trial. Ann Surg 2005; 242:302-11.

20. Thompson JF, Hunt JA, Culjak G, et al. Popliteal lymph node metastasis from primary cutaneous melanoma. Eur J Surg Oncol 2000;26:172-6.
21. Menes TS, Schachter J, Steinmetz AP, et al. Lymphatic drainage to the popliteal basin in distal lower extremity malignant melanoma. Arch Surg 2004;139:1002-6.

22. Kim HY, Chang H, Minn KW. Popliteal lymph node dissection in lower extremity malignant melanoma. J Korean Soc Plast Reconstr Surg 2009;36:485-8. 